\title{
The diagnostic role of AIM2 in Kawasaki disease
}

\section{Zhenquan Wang ${ }^{1}$, Qiaoyu Wang ${ }^{1}$, Jiahui Jin $^{1}$, Xing Rong ${ }^{1}$, Tingting $\mathrm{Wu}^{1}$, Huixian Qiu ${ }^{1}$, and Rongzhou $\mathrm{Wu}^{1}$}

${ }^{1}$ Wenzhou Medical University Second Affiliated Hospital

May 7, 2020

\begin{abstract}
Background:Kawasaki disease, a systemic vasculitis in children, may bring serious complications. However, the etiology of KD remains unclear. AIM2, an intracellular receptor, plays a vital role during the infection caused by a variety of pathogens. However, its role in KD remains unclear. The principal aim of the present research is to concentrate on the relation between AIM2 and KD. Methods: We detected the serum level of AIM2, IL-18 and IL-1 $\beta$ in all subjects by ELISA. The conventional inflammatory indices were detected in all subjects, such as WBC, HB, CRP and so on. Results: The serum concentrations of AIM2, IL-18 and IL-1 $\beta$ were notably increased in the KD group compared to the febrile group and healthy group, respectively. And the three indicators in the KD patients were greatly reduced after interpreted with IVIG. Furthermore, the expressions of IL-18 and IL-1 $\beta$ were positively correlated with AIM2. Meanwhile,the cutoff value of serum AIM2 level for the diagnosis of $\mathrm{KD}$ was $541.90 \mathrm{ng} / \mathrm{L}$ with the specificity of $60 \%$ and sensitivity of $92.5 \%$,compared to the febrile controls. And the area under curve (AUC) of AIM2 was 0.771. And no difference was observed in the CALs group when compared without the CALs group. Conclusions: The serum AIM2, IL-18 and IL- $1 \beta$ might play a critical role during the progress of KD. AIM2 can be considered as a candidate indicator for Kawasaki disease diagnosis. Keywords: AIM2 · IL-18 IL-1 $\beta$. Kawasaki disease · Intravenous immunoglobulin ·coronary artery lesions
\end{abstract}

\section{Hosted file}

mymanuscrip.doc available at https://authorea.com/users/319392/articles/449105-thediagnostic-role-of-aim2-in-kawasaki-disease
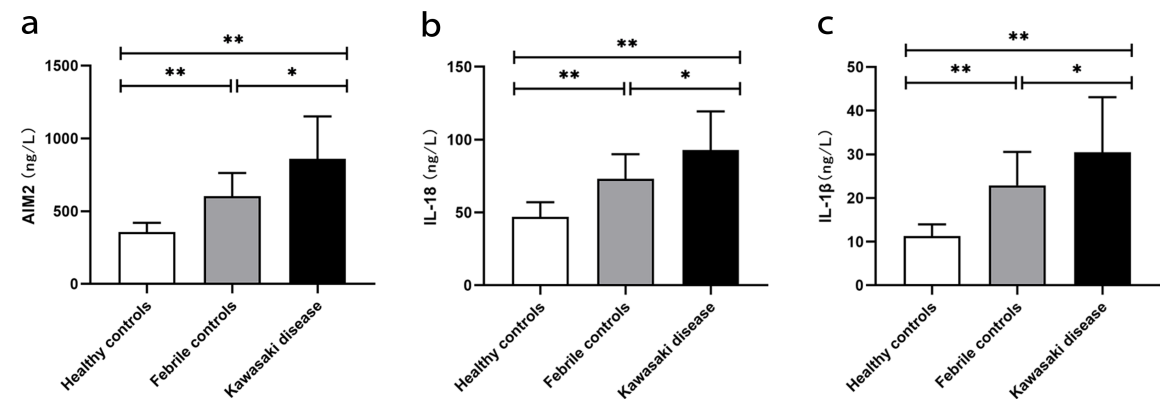

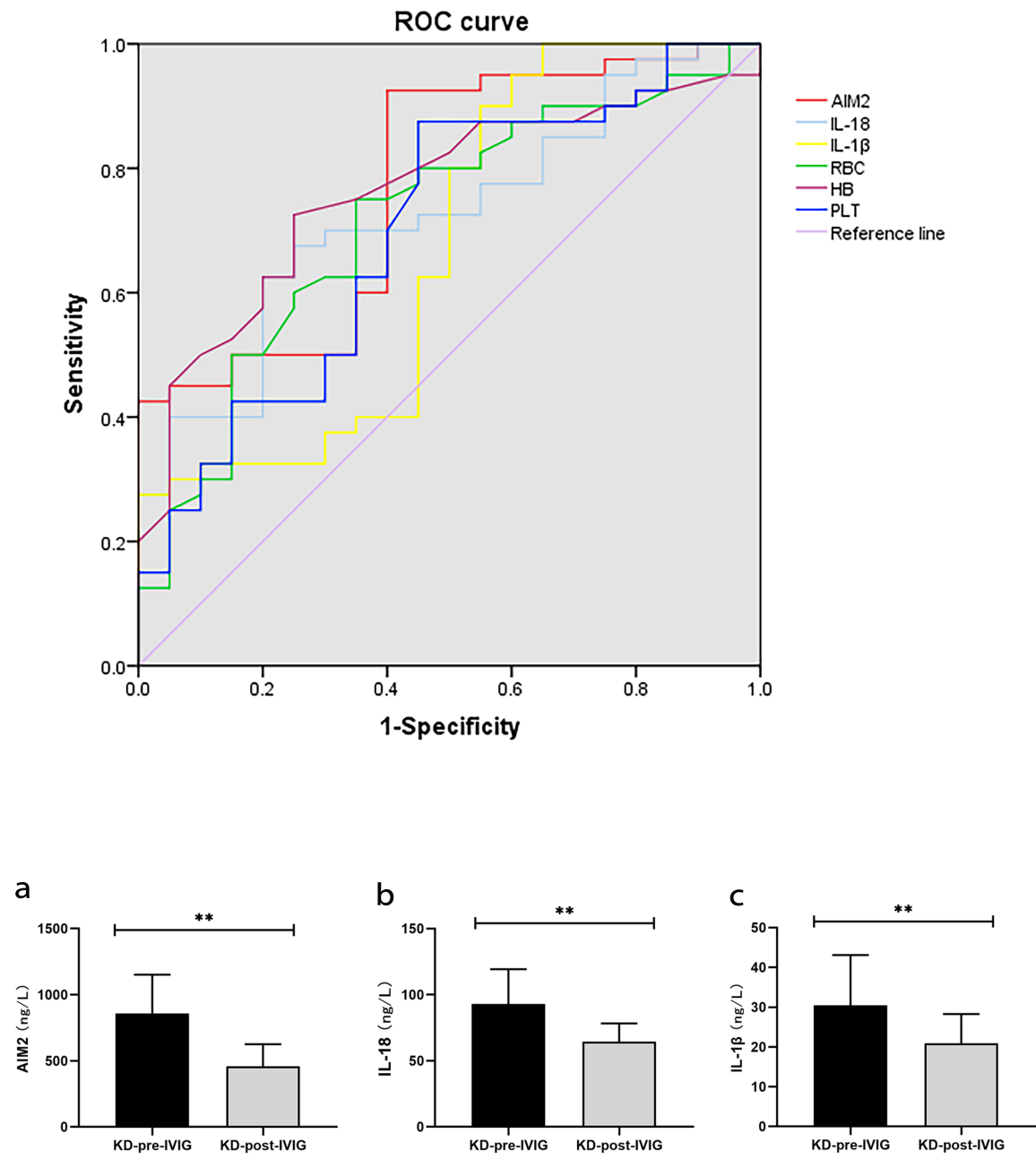